\title{
Morphometric Nerve Fiber Analysis and Aging Process of the Human Inferior Alveolar Nerve
}

\author{
By
Narumi SAGARA, Hiroshi MORIYAMA, Yasushi MIYAUCHI, Hiroaki TANI and Noboru GOTO \\ Department of Anatomy, Showa University School of Medicine, 5-8, Hatanodai 1, Shinagawa-ku, Tokyo 142-8555, \\ Japan \\ - Received for Publication, February 6, 2001 -

\begin{abstract}
Key Words: Inferior alveolar nerve, Morphometry, Aging process, Axon, LPH staining method
Summary: We studied morphometric nerve fiber analysis and the aging process of the inferior alveolar nerve (IAN). Human IANs were resected at the mandibular foramen. The preparation of sections involved fixation, washing, dehydration, embedding, sectioning and staining as described in our previous reports. We estimated the total number of myelinated axons in the IAN being average 26,200 , the transverse area of those being average $37.1 \mathrm{\mu m}^{2}$, the average perimeter of those being $23.0 \mu \mathrm{m}$, the average circularity ratio of those being 0.85 , respectively. According to these results, the IAN did not demonstrate notable age-relation change in any measured item. The IAN differed from not only our previous data on the human motor peripheral nerves but also those on the human sensory peripheral nerves. With regard to the aging process, the IAN indicated morphological features compared with other peripheral nerves.
\end{abstract}

The inferior alveolar nerve is the nerve most routinely anesthetized by dental injection during almost every restorative and surgical procedure in the mandible. However, details of this nerve and its aging process have not been included in textbook descriptions. This study precisely investigated this nerve to clarify these issues.

\section{Material and Methods}

Human inferior alveolar nerves (IANs) were resected at the mandibular foramen. Such material was obtained from 30 cadavers ( 8 males and $22 \mathrm{fe}$ males) aged 59-92 years (average age: 78.8 years). The causes of death did not directly or indirectly influence the nervous system, so the inferior alveolar nerves were considered normal. The preparation of sections involved fixation, washing, dehydration, embedding, sectioning and staining as described in our previous reports ${ }^{3,11}$.

\section{Morphometry}

The systemic sampling method was employed to measure axons. A sampling site at the center of the IAN was selected, as shown in Fig. 1. Highly enlarged images of areas in a square eyepiece grid (the area covered was $0.004 \mathrm{~mm}^{2}$ ) were selected to count the myelinated axons and to measure the transverse area and perimeter of axons. We employed (a) a microscope (BH2, Olympus, Tokyo, Japan), equipped with a drawing tube (BH2-DA, Olympus, Tokyo, Japan), (b) a digitizer (Crystizer $\alpha$ KC 3300, Graphtec, Yokohama, Japan) and (c) a computer (PC-9801 VX2, NEC, Tokyo, Japan) with an analyzing system software (original programming) for storing data on-line, calculation and statistical analysis. Regression analysis was performed between age and each of the number of fibers, the circularity ratio (CR) of fibers, axonal area and the axonal perimeter. Circularity ratios (CRs) were calculated as follows: $C R=4 \pi \mathrm{A} / \mathrm{L}^{2}(\mathrm{~A}=$ area in $\mathrm{mm}^{2} ; \mathrm{L}=$ perimeter in $\mathrm{mm}$ )

Correspondence to Prof. Noboru Goto MD, Department of Anatomy, Showa University School of Medicine. 5-8, Hatanodai 1, Shinagawa-ku, Tokyo 142-8555, Japan 


\section{Results}

\section{Number of Myelinated Axons}

We estimated the total number of myelinated axons in the IAN as ranging between 5,328 and 49,328 (average 26,200). The data for all cases are listed in Table 1 . The myelinated nerve fibers appeared as a blue-green myelin sheath surrounding a dark purple or black axon (Fig. 2). According to our data, the number of myelinated axons did not show a marked change with age $(r=0.014$; Fig. 3$)$.

\section{Transverse Area of Myelinated Axons}

The transverse area of myelinated axons in the human IAN ranged from 18.3 to 79.8 (average 37.1) $\mu \mathrm{m}^{2}$ (Table 1). According to our data, the transverse area of myelinated axons did not show a marked change with age ( $r=0.108$; Fig. 4$)$.

\section{Perimeter of Myelinated Axons}

The average perimeter of myelinated axons in the human IAN was $23.0 \mu \mathrm{m}$ (ranging from 15.9 to 33.0) (Table 1). According to our data, the perimeter of myelinated axons did not show a marked change with age ( $r=0.091$; Fig. 5).

\section{CR of Myelinated Axons}

The average $\mathrm{CR}$ of myelinated axons in the human IAN was 0.85 (ranging from 0.64 to 0.92 ;

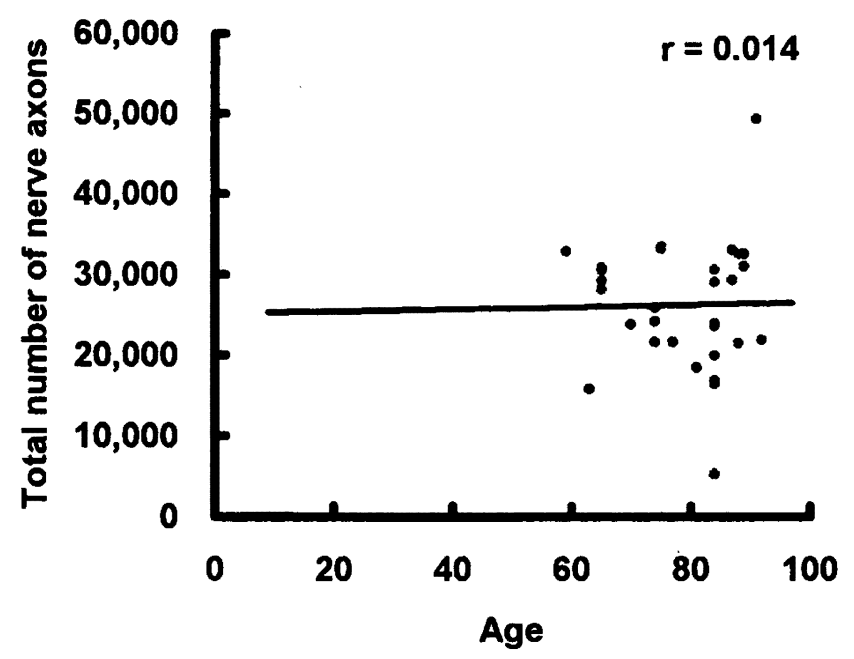

Fig. 3. Scatter diagram of the human inferior alveolar nerve showing a regression analysis between the total number of myelinated axons and age.

Table 1. Myelinated fiber analysis of the human inferior alveolar nerve

\begin{tabular}{|c|c|c|c|c|c|c|}
\hline Sex & Side & Age & Total number & Area $\left(\mu \mathrm{m}^{2}\right)$ & Perimeter $(\mu \mathrm{m})$ & CR \\
\hline $\mathrm{F}$ & L & 59 & 32,928 & 21.9 & 18.3 & 0.82 \\
\hline $\mathbf{M}$ & $\mathbf{R}$ & 63 & 15,784 & 33.8 & 21.7 & 0.88 \\
\hline $\mathbf{M}$ & $\mathrm{L}$ & 65 & 29,251 & 38.1 & 23.8 & 0.83 \\
\hline $\mathbf{M}$ & $\mathbf{R}$ & 65 & 28,125 & 32.9 & 21.1 & 0.89 \\
\hline $\mathrm{F}$ & $\mathbf{L}$ & 65 & 30,861 & 48.1 & 25.9 & 0.87 \\
\hline$F$ & $\mathrm{R}$ & 65 & 30,559 & 36.5 & 26.8 & 0.64 \\
\hline $\mathbf{M}$ & $\mathbf{L}$ & 70 & 23,773 & 18.3 & 15.9 & 0.88 \\
\hline $\mathbf{M}$ & $\mathbf{R}$ & 74 & 24,154 & 79.8 & 32.9 & 0.89 \\
\hline $\mathbf{F}$ & $\mathbf{R}$ & 74 & 25,834 & 31.9 & 21.3 & 0.86 \\
\hline $\mathbf{F}$ & $\mathbf{L}$ & 74 & 21,630 & 47.9 & 25.5 & 0.88 \\
\hline $\mathbf{F}$ & $\mathbf{R}$ & 75 & 33,186 & 25.2 & 18.7 & 0.89 \\
\hline $\mathbf{M}$ & $\mathbf{R}$ & 75 & 33,489 & 42.2 & 26.3 & 0.75 \\
\hline $\mathbf{M}$ & $\mathbf{R}$ & 77 & 21,615 & 38.2 & 24.2 & 0.82 \\
\hline $\mathbf{F}$ & $\mathrm{L}$ & 81 & 18,440 & 32.8 & 21.6 & 0.86 \\
\hline F & $\mathrm{L}$ & 84 & 29,004 & 31.3 & 21.4 & 0.84 \\
\hline F & $\mathbf{R}$ & 84 & 19,878 & 45.3 & 24.9 & 0.88 \\
\hline $\mathrm{F}$ & $\mathbf{R}$ & 84 & 30,550 & 33.9 & 21.9 & 0.85 \\
\hline F & $\mathbf{L}$ & 84 & 16,408 & 32.6 & 21.5 & 0.84 \\
\hline F & $\mathrm{L}$ & 84 & 5,328 & 32.4 & 21.2 & 0.87 \\
\hline F & $\mathrm{L}$ & 84 & 23,522 & 32.1 & 21.4 & 0.85 \\
\hline $\mathrm{F}$ & $\mathrm{L}$ & 84 & 23,884 & 28.3 & 19.3 & 0.92 \\
\hline F & $\mathrm{L}$ & 84 & 16,814 & 27.7 & 20.4 & 0.83 \\
\hline F & $\mathrm{L}$ & 87 & 29,234 & 35.9 & 22.8 & 0.85 \\
\hline $\mathrm{F}$ & $\mathrm{R}$ & 87 & 33,026 & 32.3 & 21.4 & 0.83 \\
\hline$F$ & $\mathrm{R}$ & 88 & 21,435 & 41.3 & 25.5 & 0.79 \\
\hline $\mathrm{F}$ & $\mathrm{L}$ & 88 & 32,510 & 35.4 & 22.8 & 0.82 \\
\hline$F$ & $\mathrm{R}$ & 89 & 31,006 & 33.6 & 22.7 & 0.82 \\
\hline $\mathrm{F}$ & $\mathrm{L}$ & 89 & 32,555 & 44.2 & 25.1 & 0.86 \\
\hline $\mathbf{M}$ & $\mathrm{L}$ & 91 & 49,328 & 54.7 & 27.4 & 0.85 \\
\hline $\mathrm{F}$ & $\mathrm{R}$ & 92 & 21,888 & 43.6 & 24.6 & 0.88 \\
\hline
\end{tabular}




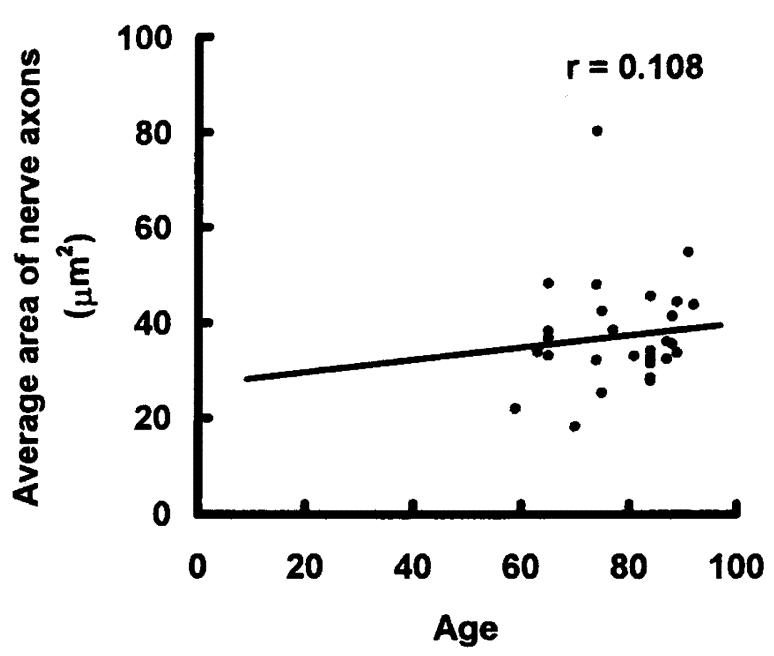

Fig. 4. Scatter diagram of the human inferior alveolar nerve showing a regression analysis between the average transverse area of myelinated axons and age.

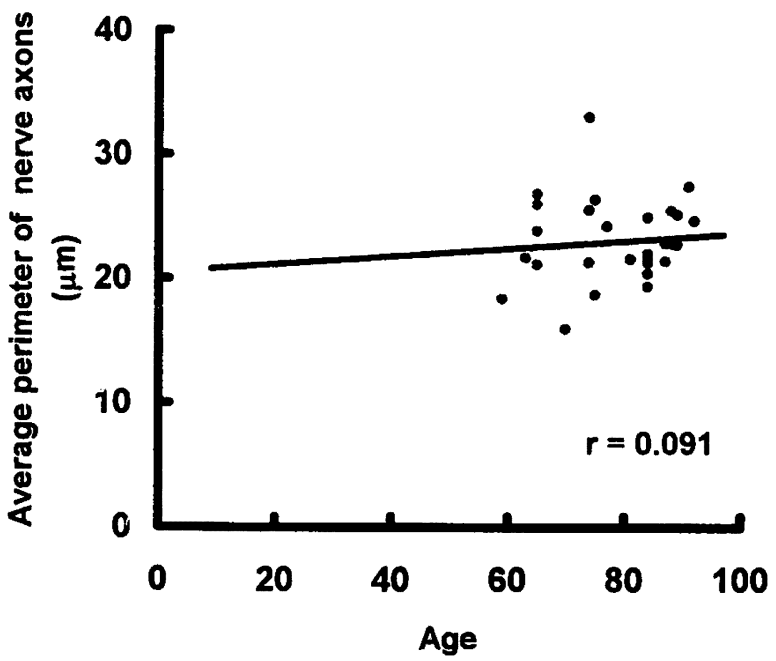

Fig. 5. Scatter diagram of the human inferior alveolar nerve showing a regression analysis between the average perimeter of myelinated axons and age.

Table 1). According to our data, the CR of myelinated axons did not show a marked change with age $(r=0.128$; Fig. 6$)$.

\section{Discussion}

There are many reports available on the morphometric analysis of nerve fibers in the animal inferior alveolar nerve (IAN), but there are few reports on that in humans ${ }^{4-7,13)}$. And there are few reports available on the morphometric analysis of nerve fibers in the human IAN especially with ag-

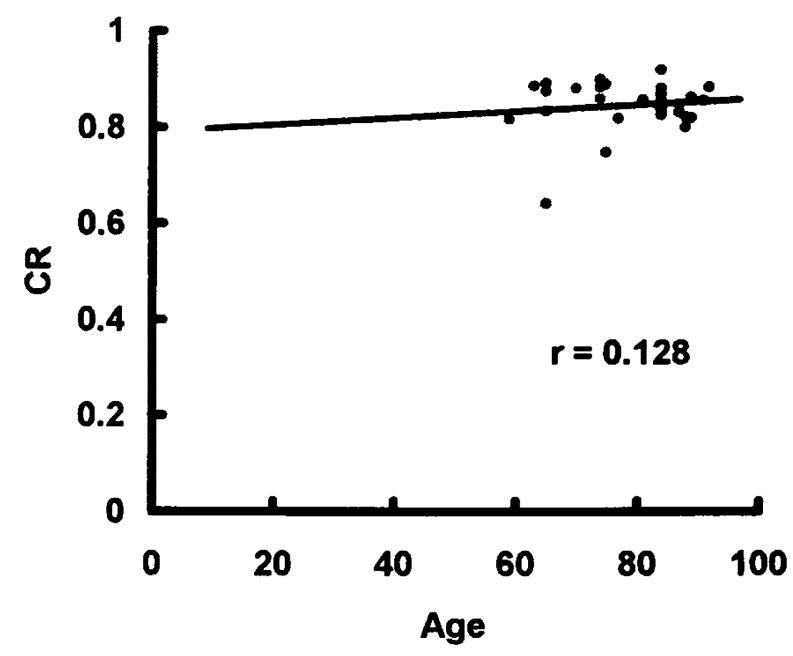

Fig. 6. Scatter diagram of the human inferior alveolar nerve showing a regression analysis between the average CR (circularity ratio) of myelinated axons and age.

ing process. The reason may be due to the lack of a discriminative staining method for nerve fibers, and a lack of modern methods for morphometric analyses. Even the electron microscope has contributed little to the quantitative analysis of nerve fibers except for that of the cat IAN $^{1,8,9)}$. Our own staining method (LPH staining method) may be ideal for morphometric research on the nervous system because of its clear discrimination of structures, while inducing minimal shrinkage (only about $10 \%$ in length).

There have been a few reports on the number of fibers in the human IAN. According to these reports, total numbers of myelinated fibers in the human IAN were 15,424 (ranged from 1,307 to 21,220 Heasman et al. $)^{6)}, 15,513$ (Rood) ${ }^{13)}, 19,514$ (ranged from 1,307 to 28,078 Heasman et al. $)^{7)}$. Our study showed similar results, with a range of 5,328 to 49,328 (average 26,200).

We have studied morphometric nerve fiber analysis and the aging process of the various human peripheral nerves. These data were compared with the present study on the IAN. With regard to the number of myelinated axons, various studies showed that the number decreased with age in the oculomotor $^{15)}$, maxillary ${ }^{10)}$, abducent ${ }^{14)}$, facial $^{2)}$ and deep peroneal ${ }^{17)}$ nerves, while that in the vestibular $^{12)}$, lesser splanchnic ${ }^{16)}$ and IAN did not. Next, various studies showed that the transverse area and the average perimeter of myelinated axons decreased with age in the maxillary ${ }^{10}$, vestibular ${ }^{12)}$, deep peroneal ${ }^{17)}$ and lesser splanchnic ${ }^{16)}$, while that in the oculomotor ${ }^{15)}$, abducent ${ }^{14)}$ and facial $^{21}$ and IAN did not. 
According to our current results, the IAN did not demonstrate any notable age-relation change in all measuring items. The IAN differed from not only our previous data of the human motor peripheral nerves but also those of human sensory peripheral nerves. With regard to the aging process, the IAN indicated morphological feature comparable to those of other peripheral nerves.

\section{References}

1) Fried $\mathrm{K}$ and Hildebrand $\mathrm{C}$. Axon number and size distribution in the developing feline inferior alveolar nerve. $\mathrm{J}$ Neurological Sciences 1982; 53:169-180.

2) Fujii $M$ and Goto $N$. Nerve fibre analysis of the facial nerve. Ann Otol Rhinol Laryngol 1989; 98:732-735.

3) Goto N. Discriminative staining methods for the nervous system: Luxol fast blue-periodic acid Schiff-hematoxylin triple stain and subsidiary staining methods. Stain Technol 1987; 62:305-313.

4) Heasman PA and Beynon ADG. Myelinated fibre diameters of human inferior alveolar nerves. Archs Oral Biol 1986; 31:785-787.

5) Heasman PA and Beynon ADG. Clinical considerations from axonmyelin relationship in human inferior alveolar nerve. Int J Oral Maxillofac Surg 1987; 16:346-351.

6) Heasman PA and Beynon ADG. Myelinated axon counts of human inferior alveolar nerves. J Anat 1987; 151:51-56.

7) Heasman PA and Beynon ADG. Quantitative analysis of inferior alveolar nerve fibre counts in man. J Dent Res 1983; 62:418.
8) Holland GR. Fibre numbers and sizes in the inferior alveolar nerve of the cat. J Anat 1978; 127:343-352.

9) Holland GR and Robinson PP. The number and size of axons central and peripheral to inferior alveolar nerve injuries in the cat. J Anat 1990; 173:129-137.

10) Kaneko $Y$, Duan J, Goto $N$ and Nagai $Y$. Nerve fiber analysis and agerelated changes of the human maxillary nerves. Showa Igakkai Zasshi 1999; 59:145-150 (in Japanese).

11) Moriyama $\mathrm{H}$, Shimada $\mathrm{K}$ and Goto N. Morphometric analysis of neurons in ganglia: Geniculate, submandibular, cervical spinal and superior cervical. Okajimas Folia Anat Jpn 1995; 72:185-190.

12) Nagai $Y$, Goto N, Goto J, Kaneko $Y$ and Suzaki H. Morphometric nerve fiber analysis and aging process of the human vestibular nerve. Okajimas Folia Anat Jpn 1999; 76:95-100.

13) Rood JP. The diameters and internodal lengths of the myelinated fibres in human inferior alveolar nerve. J Dent 1978; 6:311-315.

14) Sawabe $Y$, Matsumoto $K$, Goto N, Otsuka $N$ and Kobayashi N. Morphometric nerve fiber analysis and aging process of the human abducent nerve. Okajimas Folia Anat Jpn 1998; 74:337-344.

15) Takeshita $Y$, Matsumoto $K$, Goto $N$ and Shibata $M$. Nerve fiber analysis and aging process of the human oculomotor nerve. Showa Univ J Med Sci 1996; 8:55-61.

16) Tang W, Goto N, Tanaka J and Otsuka N. Myelinated nerve fibre analysis of the human small splanchnic nerve. Okajimas Folia Anat Jpn 1997; 74:93-98.

17) Yanagisawa $K$, Goto $N$, Kimura $T$ and Tanaka J. Nerve fiber analysis on aging process of deep peroneal nerve in man. Showa Igakkai Zasshi 1994; 54:249-254 (in Japanese). 


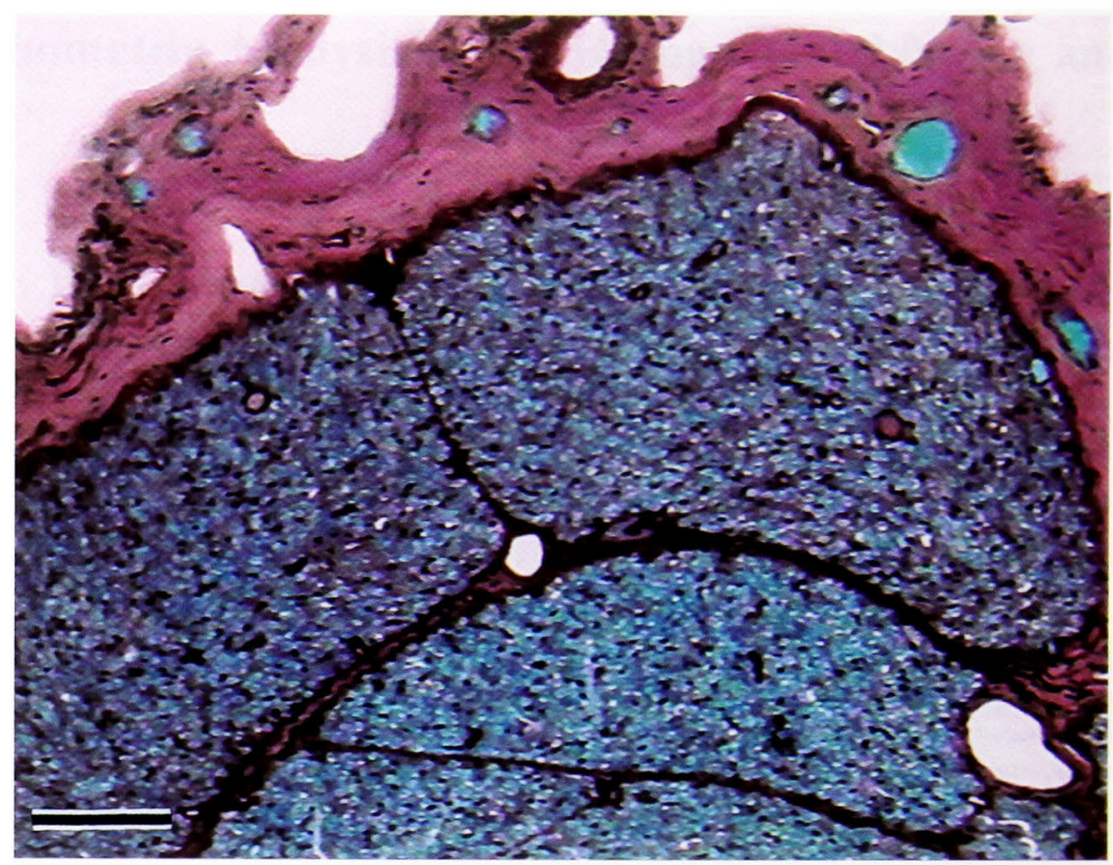

Fig. 1. A low-power view of the inferior alveolar nerve from a 63-year-old man, LPH stain. Scale bar $=100 \mu \mathrm{m}$.

\section{Explanation of Figures}

\section{Plate I}

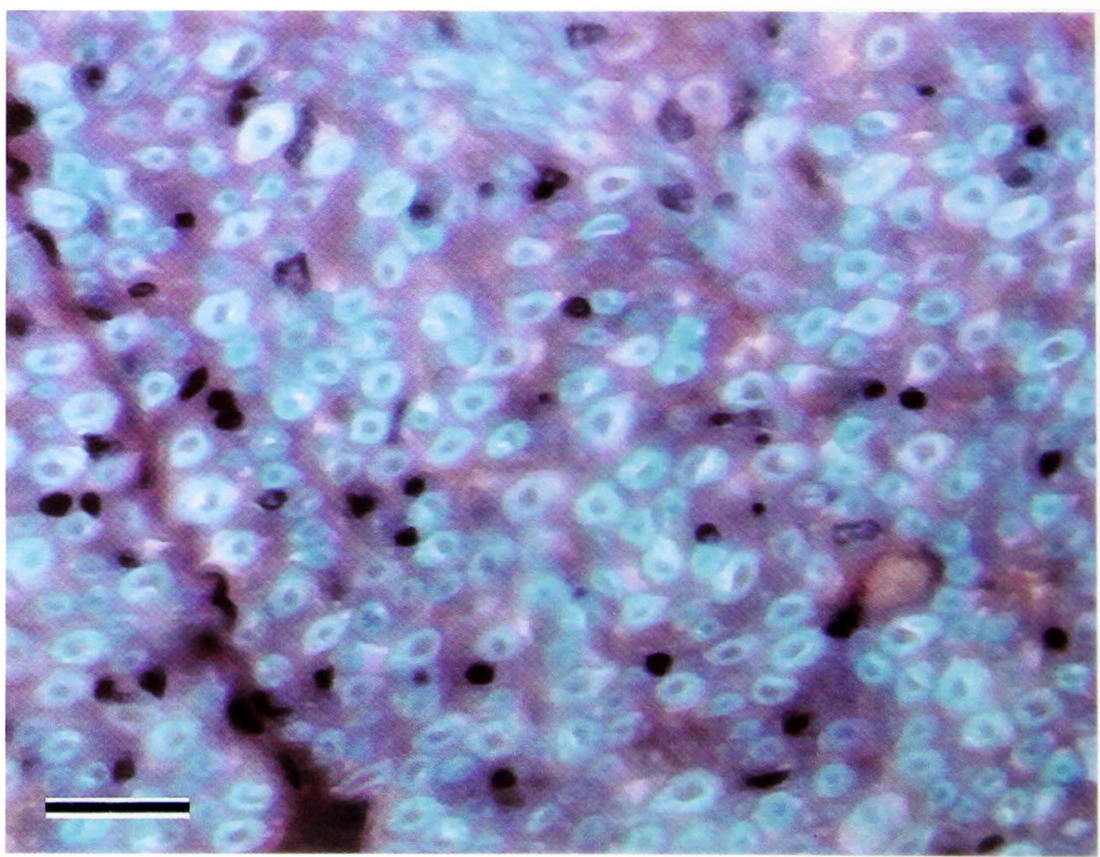

Fig. 2. High-power view of the inferior alveolar nerve from a 63-year-old man. LPH stain. Axons were stained in dark purple or black, and surrounded by myelin sheath. Scale bar $=20 \mu \mathrm{m}$. 